
\title{
$\begin{array}{ll}\text { Research Square } & \begin{array}{l}\text { Preprints are preliminary reports that have not undergone peer review. } \\ \text { They should not be considered conclusive, used to inform clinical practice, } \\ \text { or referenced by the media as validated information. }\end{array}\end{array}$
}

\section{Comparative Assessment of Modified Bentonites as Retardation Barrier: Adsorption Performance and Characterization}

\section{Dongdong Guo ( $\square$ guodongdong18@mails.ucas.ac.cn )}

Wuhan Institute of Rock and Soil Mechanics Chinese Academy of Sciences https://orcid.org/0000-0002-3357-1454

Yong Wan

Chinese Academy of Sciences Wuhan Institute of Rock and Soil Mechanics

Jiangshan Li

Institute of Rock and Soil Mechanics, Chinese Academy of Sciences

Ruiqi Liu

Wuhan Institute of Rock and Soil Mechanics, Chinese Academy of Sciences

Lei Liu

Wuhan Institute of Rock and Soil Mechanics, Chinese Academy of Sciences

Qiang Xue

Wuhan Institute of Rock and Soil Mechanics, Chinese Academy of Sciences

\section{Research Article}

Keywords: Bentonite, Modification, Heavy metal, Organic contaminant, Adsorption

Posted Date: January 4th, 2022

DOI: https://doi.org/10.21203/rs.3.rs-1047398/v1

License: (c) (i) This work is licensed under a Creative Commons Attribution 4.0 International License. Read Full License 


\section{Abstract}

Modified bentonites for anti-seepage system application has been attracting global attentions. At the same time, the performances of modified bentonite containing retardation barrier exposed to organic-heavy metal pollutants have not been fully reported. In this study, the adsorption performances (one of the key evaluation indicators of retardation barrier) of nine kinds of commonly used modified bentonites on multiple contaminants were comparatively investigated. The $X-$ ray diffraction (XRD) and Fourier transform infrared spectroscopy (FTIR) analyses were also performed to unravel the adsorption mechanisms. Results show that the adsorption of modified bentonites on phenol and $\mathrm{Pb}$ (II) follows the order of $\mathrm{SB}-16>\mathrm{PVA}>\mathrm{CTAB}>\mathrm{APAM}>\mathrm{CTAB}+\mathrm{PAC}>\mathrm{PAC}>\mathrm{CPAM}>\mathrm{CTAB}+\mathrm{PAC}>\mathrm{CTAB}+\mathrm{CPAM}+\mathrm{APAM}$. Among all the samples, the bentonite modified with SB-16 showed the highest adsorption capacities for phenol and $\mathrm{Pb}(\mathrm{II})$. $\mathrm{The}$ surfactant molecules inserted in the interlayer space of montmorillonite increase the substrate spacing, which changes the structural properties of the bentonite from hydrophilic to hydrophobic and increases the adsorption of organic contaminants. On the other hand, the polymer has functional groups such as hydroxyl and carboxyl that can form a spatial three-dimensional cross-linking structure on the bentonite surface, providing more adsorption sites for heavy metal ions. These findings indicate the potential industrial applications of modified bentonite in a contaminant barrier system.

\section{Highlights}

- The changes in structure of nine kinds of modified bentonites were obtained.

- The surfactant intercalation of bentonite resulted in very effective adsorption for organic contaminants.

- The polymer-modified bentonite with a cross-linking structure posed strong adsorption on heavy metal ions.

- The adsorption performance of modified bentonites on both phenol and $\mathrm{Pb}(\mathrm{II})$ varied.

\section{Introduction}

Urban industrial contaminated sites are characterized by complex contamination components, severe pollution, and considerable impact depth, which have a serious impact on human health, ecological environment, and groundwater structure. This problem has also seriously hindered the national planning of new urbanization and the safe development of underground space (Abuabdou et al., 2020; Xue et al., 2020). Nevertheless, the leaching of organic compounds and heavy metals from the contaminated sites, leads to the spread of contaminants to the adjacent soil and groundwater due to dissolution process, erosion, or others. The leaching of pollutants from contaminated sites not only causes serious environmental problems, but also threatens human health. Typical contaminants present in the adjacent soil or water of urban industrial contaminated sites include organic solutes such as volatile organic compounds (VOCs), and heavy metals such as lead, zinc, and cadmium, all of which are contaminants that need to be addressed and treated urgently. (Huang et al., 2021; Sonne et al., 2018; Ying et al., 2017).

In order to control the risk of urban industrial contaminated sites, bentonite-based impermeable materials (e.g., composite geotechnical liner (GCL), soil-bentonite (SB) barrier, and compacted sand-bentonite mixture) are widely used in groundwater seepage and landfill barrier systems due to the high swelling capacity and very low permeability (Sun et al., 2021; J. Scalia IV, 2018; Rowe, 2005). However, researches have shown that the leaching of pollutants from contaminated sites can cause structural failure of bentonite-based barrier structures (Xu et al., 2019; TOUZEFOLTZ et al., 2006; Xue et al., 2012; Vadlamudi and Mishra, 2018; Gupt et al., 2020; Zhang and Qiu, 2010). Changes in engineering properties due to structural failure of bentonite impermeable materials can be attributed to: (1) contraction of the diffusion double layer of bentonite particles due to increased metal ion concentration in the pore water (Benson and Meer, 2009; Li et al., 2013; Wan et al., 2020); (2) exhibit limited intrinsic adsorption capacity for organic solutes due to lacking of an appreciable amount of organic carbon (Goodarzi et al., 2016; Malusis et al., 2010; Mendes et al., 2013). 
Therefore, the modification of bentonite to improve its chemical compatibility and service performance in complex environments is the focus of the current study.

The current study on modified bentonite in the geotechnical field mainly focuses on permeability and swelling (Fan et al., 2014; Keramatikerman et al., 2017; Xu et al., 2019; Xu et al., 2016; Yang et al., 2017). For the application of impermeability of contaminated sites, the hindrance of contaminants is also an important index to be examined. The retardation factor is proportional to the adsorption constant, so it is necessary to study the adsorption effect of modified bentonite on contaminants (Chen et al., 2020; Yang et al., 2017; Yang et al., 2019). For the fast flowrate scenario (e.g., permeable reactive barriers with sorptivity), the retardation factor can be estimated from the distribution coefficient $K_{\mathrm{d}}$ (Freeze and Cherry, 1979). Hence, the adsorption constants obtained from the adsorption experiments conducted in this paper can indirectly show the barrier performance of bentonite against contaminants.

$$
R=1+\frac{\rho_{b}}{n} \cdot K_{\mathrm{d}}
$$

1

where $\rho_{b}=$ porous media bulk density $\left(\mathrm{g} / \mathrm{cm}^{3}\right) ; n=$ effective porosity of the media at saturation (\%).

In the last decade, many modified bentonites have been developed, focusing on improving the adsorption capacity or reducing the permeability of heavy metal contaminants. The traditional organic modifier in the environmental geotechnical field is the quaternary ammonium cation (QAC), which is also the most widely used organic cation. Usually, organic cations can be inserted into the interlayer space of montmorillonite through cation exchange reactions (Slade and Gates, 2004; Zhu et al., 2019). The interacting cations are then bound to the surface of montmorillonite mainly through electrostatic interactions (Xi et al., 2005). Through hydrophobic interactions between the embedded surfactant cations and the alkyl chains of the external surfactant molecules, other surfactant molecules can be co-adsorbed into the interlayer space of montmorillonite (Sun et al., 2013; Zhou et al., 2019). It was shown that the synthetically modified bentonite could effectively remove hydrophobic organic contaminants from water, but its permeability coefficient in inorganic solutions increased (Chen et al., 2020). Hence, some scholars have improved the modification of bentonite by adding hydrophilic polymers to form a three-dimensional cross-linking structure among the bentonite particles to enhance the adsorption of heavy metal ions (Fan et al., 2020, Chen et al., 2019). Although organic modified bentonite is attracting more and more attention in various fields, adsorption properties and applicable engineering conditions of the synthetically modified bentonite in the previous literature are difficult to be compared due to the various raw materials. At present, the adsorption performances of modified bentonites prepared by different modification methods have not been compared and evaluated.

In summary, amounts of exciting researches on the modification of bentonite have been investigated in the past decades (He et al., 2014; Zhao et al., 2017), but there are still many problems that deserve further exploration. First, most studies are limited to a single type of contaminant, and there is a lacking of experimental research on multiple contaminants that are closer to actual engineering conditions. In addition, the current investigations don't have an evaluation on the performance of different types of modified bentonites, and hence cannot provide valuable recommendations for the selection of engineering applications. Finally, the microscopic mechanism of removing contaminants by various types of composite modified bentonite has not been deeply compared and explored.

Therefore, this paper mainly investigated the effects of multiple contaminants on the adsorption performance of different kinds of modified bentonites and explained in relation to the physicochemical characterization. Also, batch adsorption experiments and physicochemical property tests were conducted to reveal the mechanism of heavy metalorganic contaminant-bentonite-polymer interactions. Calcium-based bentonite exhibits a lower swelling index than 
sodium-based bentonites, so they are rarely directly used in engineering. In view of the extensive storage of calciumbased bentonite in the world, its modification and application prospects are promising (Fan et al., 2014; Yang et al., 2017). The results of this study can be used to optimize the doping of organic modifiers to optimize the engineering properties of calcium bentonite, and provide a theoretical basis for the broad application of this high-performance modified bentonite in engineering and contaminant control.

\section{Materials And Methods}

\subsection{Materials}

The raw calcium bentonite (Ca-B) was obtained from Shandong province (China) with the estimated cation exchange capacity (CEC) of $64 \mathrm{mmol} / 100 \mathrm{~g}$. It contains $62.68 \%$ montmorillonite, $32.65 \%$ anorthite, $2.16 \%$ quartz, and $2.51 \%$ calcite determined by X-ray diffraction (XRD) analysis. The index properties of Ca-B measured via ASTM standards are summarized in Table 1.

Table 1

Chemical compositions and index properties of Ca-

B used.

\begin{tabular}{|c|c|c|}
\hline \multicolumn{3}{|c|}{ Chemical compositions of Ca-B used } \\
\hline & $\mathrm{SiO}_{2}$ & 63.35 \\
\hline & $\mathrm{Al}_{2} \mathrm{O}_{3}$ & 63.35 \\
\hline & $\mathrm{CaO}$ & 5.44 \\
\hline & $\mathrm{MgO}$ & 4.38 \\
\hline & $\mathrm{Fe}_{2} \mathrm{O}_{3}$ & 7.50 \\
\hline & $\mathrm{K}_{2} \mathrm{O}$ & 0.974 \\
\hline & $\mathrm{Na}_{2} \mathrm{O}$ & 1.15 \\
\hline & $\mathrm{TiO}_{2}$ & 0.910 \\
\hline & $\mathrm{P}_{2} \mathrm{O}_{5}$ & 0.184 \\
\hline \multicolumn{3}{|c|}{ Index properties of Ca-B used } \\
\hline Property & Value & Method \\
\hline Swell index $(\mathrm{mL} / 2 \mathrm{~g})$ & 7 & (ASTM, 2006) \\
\hline $\mathrm{pH}$ & 8.39 & (ASTM, 2015) \\
\hline Specific gravity, $G_{S}$ & 2.74 & (ASTM, 2010) \\
\hline CEC (meq/100g) & 64 & (ASTM, 2010c) \\
\hline Liquid limit, $\mathrm{W}_{\mathrm{L}}(\%)$ & 112 & (ASTM, 2010b) \\
\hline Plastic limit, $W_{p}(\%)$ & 38 & \\
\hline
\end{tabular}

Phenol is an aromatic hydrocarbon and more hydrophilic than many common VOC pollutants, such as benzene, toluene, and trichloroethene, for its relatively high solubility, low octanol-water partition coefficient, and low organic carbon 
partition coefficient (LaGrega et al., 2010). Therefore, phenol may be considered as a conservative surrogate compound in examining the adsorption performance of modified bentonite on organic contaminants (Malusis et al., 2010; OcampoPerez et al., 2011). Also, $\mathrm{Pb}$ (II) was selected as the representative heavy metal contaminant due to the toxicity and common occurrence in contaminated groundwater at industrial contaminated sites in China (Pal et al., 2014; Wang et al., 2018; Yang et al., 2019). Analytically pure phenol and $\mathrm{Pb}\left(\mathrm{NO}_{3}\right)_{2}$ used in tests were obtained from Aldrich Chemical Co.

All modifying reagents were analytical grade and used without further purification. The 3- (N,N-dimethypalmitylammonio) propane sulfonate (SB-16) was purchased from Nanjing Robiot Co. Ltd. Cetyltrimethylammonium bromide (CTAB), poly anioniccellulose (PAC), polyvinyl alcohol (PVA), cationic polyacrylamides (CPAM), and anionic polyacrylamide (APAM) were of analytical purity, and brought from Aladdin Chemistry Co. Ltd (Aladdin Chemistry Co. Ltd, Shanghai, China). Deionized water with a resistivity of $18.1 \mathrm{M} \Omega \cdot \mathrm{cm}$ was used for all experiments.

\subsection{Methods}

\subsubsection{Synthesis of modified bentonites}

Modified bentonites were prepared through the following steps: (1) A quantitative amount of the modifier was first added to $100 \mathrm{~mL}$ of deionized water at a temperature of $70^{\circ} \mathrm{C}$ and stirred until completely dispersed and dissolved; (2) $10 \mathrm{~g}$ of bentonite was mixed in $100 \mathrm{~mL}$ of solution and the mixture was shaken in a constant temperature mechanical shaker (180 r/min) for $24 \mathrm{~h}$ at $70^{\circ} \mathrm{C}$; (3) The prepared organic bentonite was separated from the solid by centrifugation at 4000 $\mathrm{r} / \mathrm{min}$, washed several times with deionized water until no residue was detected, placed in an ultra-low temperature refrigerator for freeze-drying to reach a constant weight (24 h), crushed, and passed through a 200-mesh sieve (75 $\mu \mathrm{m})$. The specific modification methods as well as the representation are shown in Table 2 (He and Zhu, 2016; Zhu and Zhu, 2008; Du et al., 2021; Norris et al., 2018; Yang et al., 2021a; Zhu et al., 2011; Sanchez et al., 2019). 
Table 2

Modification methods for bentonites used in this study.

\begin{tabular}{|c|c|c|c|}
\hline \multirow[t]{2}{*}{ Samples Notations } & \multicolumn{2}{|c|}{ Parameters } & \multirow[t]{2}{*}{ References } \\
\hline & Modifier & Dosage & \\
\hline 0 & - & & \\
\hline $1 \mathrm{C}$ & CTAB & $100 \%$ CEC & (He and Zhu, 2016; Zhu and Zhu, 2008) \\
\hline \multirow[t]{2}{*}{$1 \mathrm{C} 4 \mathrm{P}$} & CTAB & $100 \%$ CEC & \\
\hline & PAC & $4 \% \mathrm{Wt}$ & \\
\hline \multirow[t]{2}{*}{$1 \mathrm{C} 4 \mathrm{CP}$} & CTAB & $100 \%$ CEC & (Zhu et al., 2010) \\
\hline & CPAM & $4 \% \mathrm{Wt}$ & \\
\hline \multirow[t]{3}{*}{ 1C4CP4AP } & CTAB & $100 \%$ CEC & \\
\hline & CPAM & $4 \% \mathrm{Wt}$ & \\
\hline & APAM & $4 \% \mathrm{Wt}$ & \\
\hline 4PAC & PAC & $4 \% \mathrm{Wt}$ & (Du et al., 2021; Norris et al., 2018) \\
\hline $4 \mathrm{CP}$ & CPAM & $4 \% \mathrm{Wt}$ & \\
\hline 4AP & APAM & $4 \% \mathrm{Wt}$ & \\
\hline SB-16 & SB-16 & $100 \%$ CEC & (Yang et al., 2021; Zhu et al., 2011) \\
\hline 2PVA & PVA & $2 \% \mathrm{Wt}$ & (Sanchez et al., 2019) \\
\hline
\end{tabular}

It is worth noting that the composite modified samples must be operated separately for obtaining a significant change in the interlayer structure. The bentonite was modified by intercalation with organic cations, then re-modified with polymer.

\subsubsection{Batch adsorption experiments}

The stock phenol and $\mathrm{Pb}(\mathrm{II})$ solution $(1000 \mathrm{mg} / \mathrm{L})$ was prepared by dissolving analytical grade phenol and $\mathrm{Pb}\left(\mathrm{NO}_{3}\right)_{2}$ in deionized water, and the desired concentration of $\mathrm{Pb}$ (II) solutions were obtained by diluting the stock solution. All adsorption experiments were performed by mixing a specific amount of modified bentonite with $80 \mathrm{~mL} \mathrm{Pb(II)/phenol-}$ containing solution in a $100 \mathrm{~mL}$ polyethylene tube, then shaken at $180 \mathrm{rpm}$ in a thermostatic shaker (SHA-B300).

The testing procedure for the batch sorption experiments followed the Chinese national standard GB/T 21851-2008 (SAC, 2008) and the specific admixtures described in Table 2. The solid to liquid ratio was set at 1:100 ( $/ \mathrm{g} / \mathrm{mL})$ to ensure that the adsorption capacity of the tested bentonite was between 20\% and 85\%. (Jin et al., 2016; Lee et al., 2002; Wang et al., 2010). Three parallel samples of each type of bentonite were tested to eliminate errors. $0.8 \mathrm{~g}$ samples were first suspended in $80 \mathrm{~mL} \mathrm{~Pb}(\mathrm{II})$ and phenol containing solutions (concentrations were 100, 200, 500, 800, and $1000 \mathrm{mg} / \mathrm{L}$ ) in the centrifuge tubes. These tubes sealed with parafilm were shaken in a thermostatic shaker (SHA-B300) to ensure adequate reaction at a constant speed of $180 \mathrm{rpm}$ and a temperature of $23 \pm 2^{\circ} \mathrm{C}$ over a $24 \mathrm{~h}$ equilibration time, based on the literature (Zhu et al., 2011; Zhu et al., 2010). The adsorbed samples were centrifuged by $4000 \mathrm{rpm}$ for $10 \mathrm{~min}$. The liquid specimens are stored in a refrigerator at $4^{\circ} \mathrm{C}$ for other tests, and the solid specimens are placed in an ultra-low temperature refrigerator and then freeze-dried for further characterization. The suspension was filtered through a $0.45 \mu \mathrm{m}$ aqueous filter. The $\mathrm{Pb}$ (II) concentrations were tested by inductively coupled plasma/optical emission spectrometry (ICPOES, PerkinElmer Optima 5300 DV). The phenol concentration was determined with a double-beam spectrophotometer, Shimadzu, model UV-160, at a wavelength of $271 \mathrm{~nm}$. The phenol concentration in the sample was 
estimated from the calibration curve established by the standard phenol solutions and absorbance, which was obtained using six standard phenol solutions with concentrations ranging from 10 to $60 \mathrm{mg} / \mathrm{L}$ (Ocampo-Perez et al., 2011). The amount of $\mathrm{Pb}(\mathrm{II})$ and phenol retained by modified bentonite was determined from Eq. (2):

$$
q_{\mathrm{e}}=\frac{\left(c_{0}-c_{\mathrm{e}}\right) V}{m}
$$

2

where $q_{\mathrm{e}}$ is the equilibrium adsorption quantity $(\mathrm{mg} / \mathrm{g}), c_{0}$ and $c_{\mathrm{e}}$ are the initial and equilibrium $\mathrm{Pb}(\mathrm{II})$ concentration $(\mathrm{mg} / \mathrm{L}), V$ is the solution volume $(\mathrm{L})$, and $m$ is the mass of modified bentonite $(\mathrm{g})$.

\subsubsection{Characterization of modified bentonites}

Analyses of the mineralogical compositions of the untreated bentonite and the synthesized modified bentonite samples were performed in an X-ray diffractometer Ultima IV (RIGAKU, Tokyo, Japan) using Cu $K_{\mathrm{a}}$ radiation $(\lambda=1.5406 \AA)$ generated at $40 \mathrm{kV}$ and $40 \mathrm{~mA}$, a scan range of $0.5^{\circ}-10^{\circ} 2 \theta$, and a scan speed of $1^{\circ} 2 \theta / \mathrm{min}$.

FTIR spectra in the range of 400 to $4000 \mathrm{~cm}^{-1}$ of selected solid samples were determined by the Nicolet 6700 FTIR spectrometer (Thermo Scientific)

BET surface areas of selected solid samples were measured by nitrogen adsorption at $77 \mathrm{~K}$ with an Asap 2460, Micromeritics Surface Area \& Pore Size Analyzer. Prior to $\mathrm{N}_{2}$ adsorption, the samples were degassed in a vacuum at $300^{\circ} \mathrm{C}$ for $12 \mathrm{~h}$. The specific surface area was determined by the ethylene glycol monomethyl ether method (EGME) in the laboratory compared to the BET method.

Other physical and chemical tests were performed on the samples as follows. The TOC content of bentonites was measured using a Vario TOC analyzer (Elementer, Germany). The SSA and pore volumes were tested using the Brunauer, Emmett and Teller (BET) method, with the ASAP 2460 analyzer (Micromeritics, USA). Bentonite - water mixtures were also used to determine the zeta potentials using a Nano NS-90 Zetasizer (Malvern, UK).

\section{Results And Analyses}

\subsection{Characterization of modified bentonites}

\subsubsection{Optimization of modification conditions}

The adsorption capacities on phenol and $\mathrm{Pb}(\mathrm{II})$ by different kinds of modified bentonites dosing and modification methods were compared for the first time. As shown from Figure 1, it is easy to find that the adsorption capacity of modified bentonite on phenol can be easily improved by surfactant intercalation, and no competitive adsorption between the composite contaminants was observed, presumably due to different adsorption mechanisms and no interfering effect between them.

The modification conditions can significantly affect the adsorption capacities of the modified bentonite towards contaminants (Parolo et al., 2014). First, when using surfactants for modification, the surface of the bentonite must be washed with deionized water. By measuring the adsorption of phenol and $\mathrm{Pb}$ (II) separately, the adsorption capacity of modified bentonite on heavy metal ions of the composite contaminants will significantly reduce without this step. Therefore, considering the combined effect of heavy metal-organic contaminant co-removal, the modified bentonite needs to be cleaned with deionized water during the preparation process to ensure that the hydroxyl groups $(-\mathrm{OH})$ on the 
bentonite surface are not wrapped or covered by surfactants. Second, compound modification, such as CTAB and CPAM, cannot be added together to the bentonite suspension. Therefore, surfactant intercalation followed by polymer addition must be used to achieve adsorption of the composite contaminants. The purpose is to prevent the modifiers from reacting with each other instead of binding in the raw bentonite structure. Third, the polymer must be heated and dissolved in water before adding the bentonite. Otherwise, it is difficult to form a uniformly dispersed modified environment.

The adsorption capacity of modified bentonite is greatly affected by the accumulation density of modifiers such as surfactants in the layer spacing. Research showed that more than $250 \%$ CEC would lead to a too dense accumulation of interlayer modifiers due to spatial site resistance, resulting in a reduced adsorption effect (He et al., 2014). Furthermore, $100 \%$ CEC modified bentonite is supposed to have a better adsorption performance, so this parameter was chosen for intercalation modifier in this study to evaluate different types of modified bentonite.

\subsubsection{X-ray diffraction (XRD)}

Figure 2 shows the small-angle XRD patterns of the untreated $\mathrm{Ca}-\mathrm{B}$ and different types of modified bentonite specimens. From Figure 2(a), it can be found that after the addition of organic cations, the characteristic peak centered at $5-7^{\circ}$ of $\mathrm{Ca}-\mathrm{B}$ shifts along a lower diffraction angle. According to the Bragg equation, the phenomenon that the layer spacing of montmorillonite gradually increases indicates that the modifier is effectively added into the interlayer of montmorillonite. It can be observed that the basal spacing of organic cation-intercalated bentonite is higher than Ca-B from Figure 2(a), indicating that the organic cation has been effectively intercalated into the interlayer space of montmorillonite. An asymmetric first-order reflection for concentrations higher than $60 \%$ CEC-CTAB was observed, suggesting a random interstratification of surfactant molecule in the interlayer space. It should be clear that a particular concentration is required for the ion exchange reflection to occur, and it can be found that a significant exchange occurs after $60 \%$ CECCTAB leading to a change in the interlayer, which is consistent with the findings in the literature (Zhu and Zhu, 2008; Zhu et al., 2010).

Figure 2(b) shows after the addition of polymer modified and the original bentonite layer spacing consistent indicates that no intercalation behavior occurred. Combined with previous literature analyses (Chen et al., 2020; Kuo Tian et al., 2019), this type of hydrophilic polymer can only be combined on the external surface of the bentonite by physical interaction, thus resulting in modified bentonite with functional groups on the surface. The hydroxyl and the carboxyl groups of the polymer can be combined with each other in water into hydrogen bonds, forming a three-dimensional cross-linking structure. Figure 2(c) shows that the composite modified bentonite still retains the intercalation behavior of the organic molecules. Therefore, the structure of the composite modified bentonite is also a comprehensive reflection of the two kinds of modifiers as observed by XRD, which will be further elucidated in the later sections.

Interestingly, through the detection of small-angle XRD, a slight fluctuation can be found in the $2^{\circ}$ diffraction angle position, but no one explained clearly, the peak here might be the internal microscopic pore structure of the sample, maybe in the grinding or testing process of the sample in contact with air to form an aerogel with pore structure aerogel (Ishii et al., 2013). It is initially speculated that it is caused by the artificial sample-making process, which does not affect the analysis of the mineral structure of montmorillonite, so the effect of the results of this study is not significant and can be ignored.

From Figure 2(d), the effect of modifiers (on bentonite) is very distinguishable. These modifiers can be mainly divided into two kinds, organic cations and combined with a polymer. Among them, the organic cation involved in the modified bentonite samples will occur intercalation and is not affected by polymer, the addition of $100 \%$ CEC is the best according to the previous findings as shown above, orgonic modified samples with $>100 \%$ CEC will form a larger stacking density and decrease the adsorption capacity due to the spatial site resistance. In this study, 100\% CEC was used as the best 
addition to focus on the role of modifier category, in which SB-16 has the best intercalation effect on bentonite, also it was an environmentally friendly, green, and non-hazardous biomass duality surfactant,- and CTAB was the second. The other polymer modifiers maintained the same layer spacing with the untreated bentonite, and no intercalation behavior was observed, which indicated that polymer modification might occur only on the bentonite surface (Zhou et al., 2019).

\subsubsection{Fourier Transform infrared spectroscopy (FTIR)}

Fourier transform infrared spectroscopy (FTIR) analysis can also illustrate the internal structural changes of bentonite before and after modification (Yang et al., 2021b; Yu et al., 2019; Zhu et al., 2005). Figure 3 shows the FTIR spectra of the untreated bentonite as well as various modified bentonites. There are six main characteristic peaks of bentonite, as listed below. In the spectral range below $600 \mathrm{~cm}^{-1}$, these bands are attributed to the structural octahedral and tetrahedral absorption bands in the montmorillonite lattice. The peak at $466 \mathrm{~cm}^{-1}$ can be assigned to the Si-O-Al bending vibration characteristic peak, and the peak at $519 \mathrm{~cm}^{-1}$ can be assigned to the $\mathrm{Si}-\mathrm{O}-\mathrm{Mg}$ bending vibration characteristic peak. Also, the peak at $1036 \mathrm{~cm}^{-1}$ can be designated as the $\mathrm{Si}-0-\mathrm{Si}$ stretching vibration characteristic peak. The stronger absorption peak at $3623 \mathrm{~cm}^{-1}$ is attributed to the broad, and the strong absorption peak at $3431 \mathrm{~cm}^{-1}$ is attributed to the stretching vibration characteristic absorption peak of $-\mathrm{OH}$ on the carboxyl groups of adsorbed water or polymers located between bentonite layers. If the absorption peak is sharp, it indicates that the $-\mathrm{OH}$ group in water is not connected, otherwise the $-\mathrm{OH}$ group in water has been linked. The weaker absorption peak at $1637 \mathrm{~cm}^{-1}$ is the bending vibration characteristic absorption peak of interlayer adsorbed water hydroxyl $-\mathrm{OH}$, if the characteristic absorption peak at this place is stronger, it indicates that the interlayer of bentonite contains more interlayer adsorbed water.

The FTIR characteristic peaks of natural bentonite, which did not change significantly after modification, indicates that the mineral skeleton of bentonite was not changed after modification.

Compared with the FTIR spectra of natural bentonite, it can be found that the modified bentonite shows three new characteristic peaks located at nearly $1474 \mathrm{~cm}^{-1}, 2853 \mathrm{~cm}^{-1}$, and $2926 \mathrm{~cm}^{-1}$, respectively. The peaks located at 1474 $\mathrm{cm}^{-1}$ can be attributed to the shear vibration characteristic absorption peak of $-\mathrm{CH}_{2}$ in the organic modifier molecules. Those located near $2853 \mathrm{~cm}^{-1}$ and $2926 \mathrm{~cm}^{-1}$ can be attributed to the symmetric, and antisymmetric stretching vibration characteristic peaks of sub-methyl $\mathrm{H}-\mathrm{C}-\mathrm{H}$ in the $-\mathrm{CH}_{2}$ group on the organic modifier molecule, and their presence indicates that CTAB or SB-16 was introduced into the layer space of the bentonite. With the introduction of organic cations into the interlayer, the stretching and bending vibrational characteristic absorption peaks of the interlayer adsorbed water hydroxyl $-\mathrm{OH}$ located at $3431 \mathrm{~cm}^{-1}$ and $1637 \mathrm{~cm}^{-1}$ are weakened to different degrees, indicating that the interlayer adsorbed water is greatly reduced while the hydrophobicity of the surface is significantly enhanced. The characteristic absorption peak of structural water in the lattice located at $3623 \mathrm{~cm}^{-1}$ is also very different. For the modified bentonite sample with organic cation intercalation, the absorption peak here disappears, indicating that a large amount of interlayer adsorbed water is exchanged and removed from the bentonite due to the intercalation of organic modifiers. However, the polymer-modified samples still retain this peak, and the peak position is close to the untreated bentonite, indicating that the interlayer hydrophilicity is not affected. Combined with the XRD analyses of polymermodified bentonite, it shows that the polymer introduced has no intercalation behavior.

\subsubsection{Other physico-chemistry characteristics}

Specific surface area (SSA) represents the degree of contact between the active sites of the modified clay and the contaminant, i.e., the space where the contaminant is adsorbed. The surface area is divided into internal surface area, and external surface area, where the BET method commonly used can only measure the external surface area for expansive clays, since the contraction between the layers occurs at low-temperature desorption, resulting in the inaccessibility of nitrogen molecules. In this case, it is necessary to measure the accurate specific surface area by EGME method. It is not difficult to find from Figure 4 that all modified samples involving organic cation intercalation have a 
reduced SSA, indicating that the CTAB or SB-16 molecule is mainly introduced in the mesopore space (Parolo et al., 2014). As proposed by Park et al. (2011), when the introduced molecules are densely intercalated in the structure of the bentonite, both in the interlayer spaces and interparticle pores, resulting in a reduction in the specific surface area and pore volume of the tested bentonite samples. As a result, after the bentonite sample is intercalated, the interlayer space is almost completely enclosed, which leads to the entry of nitrogen and ethylene glycol monomethyl ether being blocked by organic molecules at the edges of individual platelets.

As Figure 5 shown, the layer spacing and the total organic carbon content have a high linear correlation, and the results are also consistent with the adsorption constants of modified bentonite for organic contaminants such as phenol, indicating that the adsorption capacity of modified bentonite for organic contaminants is related to the intercalation and the total organic carbon content. TOC is often associated with a high volume of aliphatic carbon as a partitioning medium. Therefore, the sorbent-water distribution coefficient $K_{d}$, which is the slope of the linear sorption isotherm, is inherently related to TOC, which is consistent with the conclusions in the literature (Zhao et al., 2017).

As shown in Figure 6, the zeta potential (६) of clay surface is widely used to measure the stability of clay dispersions, as well as to characterize the adsorption mechanism at the solid-liquid interface. It is not difficult to find that the zeta potential has a similar trend to the adsorption constant $K_{\mathrm{F}}$, and the specific reasons and mechanism need further exploration (Zadaka et al., 2010).

\subsection{Comparison of the adsorption effect on $\mathrm{Pb}$ (II) and Phenol}

To explore the adsorption of modified bentonite on composite contaminants in batch adsorption experiments, the composite contaminants were discussed separately. The intercalation effect of surfactants on the bentonite reduced the adsorption capacity of the untreated bentonite for heavy metal ions due to the occupation of a portion of the internal surface area. Figure 7 and Table 3 show the adsorption isotherms of $\mathrm{Pb}$ (II) onto the bentonites. Natural bentonite is very remarkable for removing heavy metal ions, such as $\mathrm{Pb}(\mathrm{II})$, through ion exchange reactions, because multivalent ions are effective competitors for the clay exchange sites occupied by monovalent ions. Meanwhile, cations with smaller hydration radii for the same valent ions have a stronger hydration ability to enter the interlayer of the clay mineral (Jo et al., 2001; Kolstad et al., 2004). The adsorption of $\mathrm{Pb}$ (II), however, gradually decreased as the mass of surfactant adsorbed by the bentonite increased, as shown in Figure 7. The reason for this is that the molecular weight of organic molecules is much larger than inorganic metal ions, and so the Coulomb Force generated is greater. Therefore, the organic molecules are more easily attracted to the interlayer of bentonite than inorganic ions, thus replacing the original inorganic metal ions, such as calcium and small amounts of sodium. In this way, the interlayer domains of bentonite are hydrophobic in nature and thus cannot be replaced by inorganic ions nor do they provide adsorption sites where inorganic metal ions can be adsorbed. 
Table 3

Fitting parameters of adsorption isotherms for $\mathrm{Pb}(\mathrm{II})$.

\begin{tabular}{|c|c|c|c|c|c|c|c|c|c|c|}
\hline \multirow{3}{*}{$\begin{array}{l}\text { Samples } \\
K_{\mathrm{F}} \\
\text { Value }\end{array}$} & \multicolumn{5}{|c|}{ Freundlich isotherm } & \multicolumn{5}{|c|}{ Langmuir isotherm } \\
\hline & \multicolumn{3}{|c|}{$1 / n$} & \multirow[t]{2}{*}{$\mathrm{R}^{2}$} & \multicolumn{2}{|l|}{$q_{\mathrm{m}}$} & \multicolumn{2}{|l|}{$k$} & \multirow{2}{*}{\multicolumn{2}{|c|}{$\mathrm{R}^{2}$}} \\
\hline & $\begin{array}{l}\text { Standard } \\
\text { deviation }\end{array}$ & Value & $\begin{array}{l}\text { Standard } \\
\text { deviation }\end{array}$ & & Value & $\begin{array}{l}\text { Standard } \\
\text { deviation }\end{array}$ & Value & $\begin{array}{l}\text { Standard } \\
\text { deviation }\end{array}$ & & \\
\hline 0 & 38.086 & 5.131 & 0.132 & 0.027 & 0.911 & 77.168 & 3.064 & 2.092 & 0.369 & 0.980 \\
\hline $1 \mathrm{C}$ & 31.068 & 4.543 & 0.176 & 0.030 & 0.952 & 86.853 & 20.666 & 0.067 & 0.085 & 0.572 \\
\hline $1 \mathrm{C} 4 \mathrm{P}$ & 9.147 & 5.724 & 0.214 & 0.105 & 0.684 & 56.126 & 26.333 & 0.004 & 0.004 & 0.369 \\
\hline $1 \mathrm{C} 4 \mathrm{CP}$ & 3.140 & 1.938 & 0.356 & 0.100 & 0.851 & 44.659 & 15.403 & 0.004 & 0.004 & 0.628 \\
\hline 1C4CP4AP & 1.766 & 1.148 & 0.450 & 0.105 & 0.897 & 52.755 & 19.387 & 0.003 & 0.002 & 0.768 \\
\hline 4PAC & 15.424 & 3.167 & 0.240 & 0.038 & 0.953 & 60.851 & 7.064 & 0.096 & 0.059 & 0.821 \\
\hline $4 \mathrm{CP}$ & 11.946 & 3.617 & 0.256 & 0.057 & 0.936 & 69.136 & 14.767 & 0.012 & 0.009 & 0.753 \\
\hline $4 \mathrm{AP}$ & 22.773 & 4.353 & 0.162 & 0.031 & 0.928 & 62.218 & 7.340 & 0.894 & 0.556 & 0.777 \\
\hline SB-16 & 31.197 & 4.320 & 0.177 & 0.028 & 0.956 & 86.036 & 19.658 & 0.079 & 0.101 & 0.583 \\
\hline 2PVA & 41.264 & 5.319 & 0.135 & 0.027 & 0.922 & 82.272 & 6.731 & 3.439 & 1.261 & 0.921 \\
\hline
\end{tabular}

Table 4

Fitting parameters of adsorption isotherms for phenol.

\begin{tabular}{|c|c|c|c|}
\hline Samples & Linear isotherm & & \\
\hline$K_{\mathrm{d}}$ & & $\mathrm{R}^{2}$ & \\
\hline Value & Standard deviation & & \\
\hline 0 & - & - & - \\
\hline $1 \mathrm{C}$ & 1.750 & 0.557 & 0.997 \\
\hline $1 \mathrm{C} 4 \mathrm{P}$ & 1.749 & 1.367 & 0.983 \\
\hline $1 \mathrm{C} 4 \mathrm{CP}$ & 1.465 & 1.115 & 0.988 \\
\hline 1C4CP4AP & 0.967 & 0.831 & 0.994 \\
\hline 4PAC & - & - & - \\
\hline $4 \mathrm{CP}$ & - & - & - \\
\hline 4AP & - & - & - \\
\hline SB-16 & 9.523 & 1.215 & 0.985 \\
\hline 2PVA & - & - & - \\
\hline
\end{tabular}

The adsorption of $\mathrm{Pb}$ (II) by modified bentonite was fitted using two nonlinear models, Langmuir and Freundlich, respectively, as shown in Table 3, and it was found that the Freundlich model had a better fitting with the experimental data. From Figure 8, PVA, 1.0 CTAB and SB-16 are the three modified bentonites with relatively outstanding performance. The other modified bentonite samples with added polymers have a worse adsorption effect than the untreated bentonite. It seems that the hydrophilic polymers may easily lead to flocculation due to a large number of hydrogen bonds, which 
makes the effective surface area for contaminant adsorption decreases. The higher absolute value of the zeta potential indicates that the colloidal system is more dispersed, and the lower value means that the flocculation effect is greater. In addition, the surfactant of the intercalation layer must be cleaned with deionized water to retain the better adsorption of heavy metal ions by the bentonite itself, presumably due to a large number of functional groups on the surface of the bentonite, such as hydroxyl and carboxyl groups, which can be confirmed by FTIR, indicating that the adsorption of heavy metal ions is mainly contributed by the edges and external surface of the bentonite lamellae. However, since the content of modifier in the modified bentonite accounts for a certain mass, the montmorillonite content is relatively less than that of the untreated bentonite, so the adsorption effect of the selected modified bentonite will be greatly superior to that of the untreated bentonite under the condition that the montmorillonite content is equal.

The adsorption results for phenol satisfy the Henry linear adsorption model, and the results are shown in Figure 7. The linear adsorption isotherm curve indicates that the adsorption mechanism is mainly based on the hydrophobic partitioning of alkyl chains. The data circled in the figure show that the bentonite samples modified with different polymers have no adsorption effect on phenol in the full concentration range. The only way to adsorb phenol is organic molecular intercalation (like surfactant)intercalation, in which the adsorption effect of the samples modified with 1.0 CTAB first is basically the same, and the adsorption effect of the bisexual surfactant SB-16 on phenol is much better than the other modified bentonite samples. The above experimental data indicates that the adsorption of modified bentonite on the organic modecule can only contribute to the adsorption effect by intercalation of organic cations, and the specific adsorption mechanism is mainly hydrophobic interactions and distribution of organic phases. It is worth mentioning that SB-16 as a new green surfactant should attract greater attention and is expected to be a complete replacement of monosubstituted surfactants, not only due to its superior adsorption effect, but also because of its own adaptation to the environmental requirements of a future life as an environmentally friendly and economical green modifier.

In order to better evaluate the comprehensive performance of different types of modified bentonite, a comprehensive factor $W$ the adsorption constants of organic contaminants and heavy metal contaminants by normalization were proposed to establish a comprehensive index to describe the adsorption performance of the modified bentonites quantitatively, and the following mathematical model can be established.

$$
W=\frac{x}{x_{\max }}+\frac{y}{y_{\max }}
$$

3

Where $x$ is the $K_{\mathrm{d}}$ of different samples; $x_{\max }$ is the highest $K_{\mathrm{d}}$ among different samples; $y$ is the $K_{\mathrm{F}}$ of different samples; $y_{\max }$ is the highest $K_{\mathrm{F}}$ among different samples.

From Table 5, the PVA-modified bentonite has the highest adsorption capacity for $\mathrm{Pb}(\mathrm{II})$, and the SB-16 modified bentonite has not only the highest adsorption capacity for phenol, but also the highest combined adsorption capacity for both contaminants, with a $W$ index of 1.756 . The adsorption performance of modified bentonite on both phenol and $\mathrm{Pb}$ (II) follows the ranking as SB-16 $>$ PVA $>$ CTAB $>$ APAM $>$ CTAB + PAC $>$ PAC $>$ CPAM $>$ CTAB + CPAM $>$ CTAB + CPAM + APAM. 
Table 5

Introduction of the new assessment factor $W$ combining the adsorption constants of pollutants by normalization.

\begin{tabular}{|llllll|}
\hline Samples & $K_{\mathrm{d}}$ & $x / x_{\max }$ & $K_{\mathrm{F}}$ & $y / y_{\max }$ & $W^{*}$ \\
\hline 0 & - & 0 & 38.086 & 0.923 & 0.923 \\
\hline 1C & 1.750 & 0.184 & 31.068 & 0.753 & 0.937 \\
\hline 1C4P & 1.749 & 0.184 & 9.147 & 0.222 & 0.405 \\
\hline 1C4CP & 1.465 & 0.154 & 3.140 & 0.076 & 0.230 \\
\hline 1C4CP4AP & 0.967 & 0.102 & 1.766 & 0.043 & 0.144 \\
\hline 4PAC & - & 0 & 15.424 & 0.374 & 0.374 \\
\hline 4CP & - & 0 & 11.946 & 0.289 & 0.289 \\
\hline 4AP & - & 0 & 22.772 & 0.552 & 0.552 \\
\hline SB-16 & 9.523 & 1.000 & 31.197 & 0.756 & 1.756 \\
\hline 2PVA & - & 0 & 41.264 & 1.000 & 1.000 \\
\hline * $W=x / x_{\max }+y / y_{\max }$ & & & & \\
\hline
\end{tabular}

\section{Discussion}

\subsection{Modification mechanism of organic bentonite}

Due to the lattice substitution phenomenon of bentonite, excess negative charge is generated in its lattice. In order to maintain the charge balance of its cells, bentonite adsorbs exchangeable cations such as $\mathrm{Na}^{+}, \mathrm{Ca}^{2+}$ into its interlayer domain. When cations with higher hydration capacity are introduced, and the concentration is changed to exceed a certain threshold, the cations in the interlayers of bentonite are easily exchanged by other cations, so some inorganic or organic cations can be introduced into the layer space of bentonite. In this paper, during the reaction process of bentonite modification using surfactants, ion exchange reactions occur between organic cations and the original inorganic cations between bentonite layers, resulting in the layer spacing of bentonite and the organic phase increasing, which has a great impact on the adsorption performance of bentonite.

Generally speaking, the organic modifier enters the interlayer space of bentonite through two stages: ion exchange adsorption and partitioning mechanism. Ion-exchange adsorption refers to the process of ion exchange between the organic modifier and the exchangeable cations (such as $\mathrm{Na}^{+}, \mathrm{Ca}^{2+}$ ) in the interlayer of bentonite. The partitioning mechanism refers to when the original exchangeable cations between the layers of bentonite are replaced by organic cations. The organic cations exchanged into the interlayer of the bentonite form a micro-organic distribution phase, which continues to adsorb organic modifiers through hydrophobic interaction.

The surface functional groups of the bentonite were analyzed by FTIR, combined with XRD to analyze the possible adsorption sites. FTIR analysis was performed on the untreated bentonite and modified bentonite samples. The adsorption amounts measured with the modifiers of different addition ratios were compared with the qualitative analysis of the crystal structure and its functional groups to explore the structural changes of bentonite before and after modification and the interaction between bentonite samples and the modifiers, which was verified by batch adsorption experiments. 
In this paper, CTAB-PAC/PAM- composite modified bentonite was prepared by a stepwise method. First, the cationic surfactant CTAB underwent ionic exchange with the exchangeable cation $\mathrm{Ca}^{2+}$ in the interlayer of calcium-based bentonite. The positively charged end of the surfactant was bound to the interlayer of bentonite to obtain the surfactant single modified organic bentonite CTAB-bentonite with a bottom spacing of $1.905 \mathrm{~nm}$, indicating that the CTAB between the modified bentonite layers was arranged in a predominantly pseudo-trilayered manner. In addition, when the polymer (PAC or PAM) was further introduced into the single modified CTAB-bentonite structure, the polymer could not be adsorbed in the interlayer due to its hydrophilic structure. Observed from XRD findings, the polymer did not change the layer spacing of the modified bentonite. Also FTIR showed that the modified bentonite with the introduction of special functional groups, so the polymer should be adsorbed on the external surface or edge of the bentonite through the hydroxyl and carboxyl groups.

The surfactant molecule entered the bentonite layers by ionexchange process, and the polymer added later was bound to the surface and edges of modified bentonite lamella by intermolecular forces. However, the weak interaction between polymer and the modified bentonite leads to the elution phenomenon in practical engineering, i.e., the polymer easily dissolves from the modified bentonite, leading to the failure of barrier performance, which has been a severe engineering problem.

\subsection{Mechanisms of contaminants removal}

As can be seen from Figure 7, the natural calcium-based bentonite and polymer-modified bentonite have a weak adsorption capacity for phenol, which is due to their hydrophilic surface. While surfactants with organic cations have a positive charge and can adsorb partially deprotonated and dissociated phenol anions through electrostatic interaction, and organic bentonite has large layer spacing and good hydrophobicity to form "micro-organic distribution phase", resulting in a relatively strong organic distribution medium, so it has a better effect on adsorption and removal of phenol. The larger the layer spacing of modified bentonite, the larger the adsorption space for phenol, the more prominent the phenol removal effect of modified bentonite.

The adsorption removal of heavy metal ions is generally accomplished by interlayer cation exchange and chelation on the surface.The adsorption capacity is related to the cation exchange capacity (CEC) of bentonite and the specific surface area of bentonite. From the XRD results, it is clear that although the intercalation of surfactants leads to a larger layer spacing of bentonite, the hydrophobic effect makes it difficult for heavy metal ions to enter the interlayer domain, while the external surfaces of bentonite and surface-bound polymers contain many hydroxyl and carboxyl functional groups, which increase the adsorption sites for heavy metal ions. For the composite modified bentonite, the adsorption capacity for heavy metal ions is slightly reduced than that of the single polymer modified bentonite due to the introduction of surfactant.

The linear adsorption isotherm curve indicates that the adsorption mechanism is mainly based on the hydrophobic partitioning of alkyl chains. The magnitude of the adsorption performance of organic montmorillonite not only depends on the magnitude of the adsorption interaction, but also is closely related to its adsorption capacity.

The adsorption capacity of modified bentonite is greatly affected by the accumulation density of modifiers such as surfactants in the layer spacing. Research showed that more than $250 \%$ CEC would lead to a too dense accumulation of interlayer modifiers due to spatial site resistance, resulting in a reduced adsorption effect (He et al., 2014; Zhao et al., 2017). Furthermore, $100 \%$ CEC modified bentonite is supposed to have a better adsorption performance, so this parameter was chosen for intercalation modifier in this study to evaluate different types of modified bentonite.

Partition of organic compounds into interlayer surfactant aggregates driven by hydrophobic interactions was believed to be the predominant adsorption mechanism of organoclay loading long-chain surfactant (Johnston et al., 2012; Xu et al., 2014; Zhao and Burns, 2012). 


\section{Conclusions}

Nine types of modified and untreated bentonites were prepared and charactered in this study. The adsorption performances of modified bentonites on $\mathrm{Pb}(\mathrm{II})$ and phenol were compared and evaluated, as well as the corresponding adsorption mechanism was analyzed. The following conclusions can be drawn:

- Modification improved the adsorption effectiveness of bentonites. The PVA-modified bentonite posed the highest adsorption capacity for $\mathrm{Pb}(\mathrm{II})$, and the SB-16-modified bentonite had not only the highest adsorption capacity for phenol, but also the highest combined adsorption capacity for both contaminants. The adsorption performance of modified bentonites on both phenol and $\mathrm{Pb}(\mathrm{II})$ followed the ranking as SB-16 $>\mathrm{PVA}>\mathrm{CTAB}>\mathrm{APAM}>\mathrm{CTAB}+\mathrm{PAC}>$ $\mathrm{PAC}>\mathrm{CPAM}>\mathrm{CTAB}+\mathrm{CPAM}>\mathrm{CTAB}+\mathrm{CPAM}+\mathrm{APAM}$.

- The surfactant intercalation of the bentonite resulted in very effective adsorption of organic contaminants, and the main adsorption mechanism was the hydrophobic partitioning of alkyl chains. While the polymer formed a threedimensional cross-linking structure with abundant functional groups, such as carboxyl and hydroxyl groups, on the bentonite surface and thus had a strong adsorption effect on heavy metal ions.

\section{Declarations}

\section{Declaration of Competing Interest}

The authors declare that they have no known competing financial interests or personal relationships that could have appeared to influence the work reported in this paper.

\section{Acknowledgment}

This study was financially supported by the National Key Research and Development Program (Grant No. 2019YFC1804002), National Natural Science Foundation of China (Grant No. 5197091360 and 51625903 ), Youth Innovation Promotion Association CAS (Grant No. 2021327), and the Wuhan Science and Technology Conversion Special Project (Grant No. 2018060403011348).

\section{References}

1. Abuabdou SMA, Ahmad W, Aun NC, Bashir MJK (2020) A review of anaerobic membrane bioreactors (AnMBR) for the treatment of highly contaminated landfill leachate and biogas production: Effectiveness, limitations and future perspectives. J Clean Prod 255:120215

2. Benson CH, Meer SR (2009) Relative Abundance of Monovalent and Divalent Cations and the Impact of Desiccation on Geosynthetic Clay Liners. J Geotech GeoEnviron Eng 135(3):349-358

3. Chen Y, Liao R, Yu C, Yu X (2020) Sorption of Pb(II) on sodium polyacrylate modified bentonite. Adv Powder Technol 31(8):3274-3286

4. DOMINIJANNI A, PUMA S (2013) Coupled chemical-hydraulic-mechanical behaviour of bentonites. Géotechnique 63(3):191-205

5. Du Y, Shen S, Tian K, Yang Y (2021) Effect of Polymer Amendment on Hydraulic Conductivity of Bentonite in Calcium Chloride Solutions. J Mater Civ Eng 33(2):04020452

6. Fan RD, Du YJ, Reddy KR, Liu SY, Yang YL (2014) Compressibility and hydraulic conductivity of clayey soil mixed with calcium bentonite for slurry wall backfill: Initial assessment. Appl Clay Sci 101:119-127

7. Freeze A, Cherry J (1979) GROUNDWATER. Prentice-Hall, Inc., Englewood Cliffs, NJ 07632

Page 15/24 
8. Goodarzi AR, Najafi Fateh S, Shekary H (2016) Impact of organic pollutants on the macro and microstructure responses of Na-bentonite. Appl Clay Sci 121-122:17-28

9. Gupt CB, Bordoloi S, Sekharan S, Sarmah AK (2020) A feasibility study of Indian fly ash-bentonite as an alternative adsorbent composite to sand-bentonite mixes in landfill liner. Environ Pollut 265:114811

10. He H et al (2014) Synthesis of organoclays: A critical review and some unresolved issues. Appl Clay Sci 100:22-28

11. He S, Zhu L (2016) Durability of organobentonite-amended liner for decelerating chloroform transport. Chemosphere 149:343-350

12. Huang $X$ et al (2021) Use of self-hardening slurry for trench cutoff wall: A review. Constr Build Mater 286:122959

13. Ishii Y, Nishiwaki Y, Al-zubaidi A, Kawasaki S (2013) Pore Size Determination in Ordered Mesoporous Materials Using Powder X-ray Diffraction. J Phys Chem C 117(35):18120-18130

14. Scalia IV J, G.L.B.C (2018) Enhanced bentonites for containment of inorganic waste leachates by GCLs. Geosynthetics International

15. Jin X, Zheng M, Sarkar B, Naidu R, Chen Z (2016) Characterization of bentonite modified with humic acid for the removal of $\mathrm{Cu}$ (II) and 2,4-dichlorophenol from aqueous solution. Appl Clay Sci 134:89-94

16. Jo HY, Katsumi T, Benson CH, Edil TB (2001) Hydraulic Conductivity and Swelling of Nonprehydrated GCLs Permeated with Single-Species Salt Solutions. J Geotech GeoEnviron Eng 127(7):557-567

17. Johnston CT, Khan B, Barth EF, Chattopadhyay S, Boyd SA (2012) Nature of the Interlayer Environment in an Organoclay Optimized for the Sequestration of Dibenzo-p-dioxin. Environ Sci Technol 46(17):9584-9591

18. Keramatikerman M, Chegenizadeh A, Nikraz H (2017) An investigation into effect of sawdust treatment on permeability and compressibility of soil-bentonite slurry cut-off wall. J Clean Prod 162:1-6

19. Kolstad DC, Benson CH, Edil TB (2004) Hydraulic Conductivity and Swell of Nonprehydrated Geosynthetic Clay Liners Permeated with Multispecies Inorganic Solutions. J Geotech GeoEnviron Eng 130(12):1236-1249

20. Kuo Tian WJ, Likos, Benson CH (2019) Polymer Elution and Hydraulic Conductivity of Bentonite - Polymer Composite Geosynthetic Clay Liners. J Geotech GeoEnviron Eng 145(10):04019071

21. LaGrega MD, Buckinghaml PL, Evans JC 2010. Hazardous waste management.Waveland Press

22. Lee J, Choi J, Park J (2002) Simultaneous sorption of lead and chlorobenzene by organobentonite. Chemosphere (Oxford) 49(10):1309-1315

23. Li J, Xue Q, Wang P, Liu L (2013) Influence of leachate pollution on mechanical properties of compacted clay: A case study on behaviors and mechanisms. Eng Geol 167:128-133

24. Malusis MA, Maneval JE, Barben EJ, Shackelford CD, Daniels ER (2010) Influence of adsorption on phenol transport through soil-bentonite vertical barriers amended with activated carbon. J Contam Hydrol 116(1-4):58-72

25. Mendes MJA, Touze-Foltz N, Gardoni M, Ahari M, Mazeas L (2013) Quantification of diffusion of phenolic compounds in virgin GCL and in GCL after contact with a synthetic leachate. Geotext Geomembr 38:16-25

26. Mukherjee K, Kumar Mishra A (2020) Undrained performance of sustainable compacted sand-bentonite-glass fiber composite for landfill application. J Clean Prod 244:118662

27. Norris A, Di Emidio G, Malusis MA, Replogle M (2018) Modified bentonites for soil-bentonite cutoff wall applications with hard mix water. Appl Clay Sci 158:226-235

28. Ocampo-Perez R, Leyva-Ramos R, Mendoza-Barron J, Guerrero-Coronado RM (2011) Adsorption rate of phenol from aqueous solution onto organobentonite: Surface diffusion and kinetic models. J Colloid Interface Sci 364(1):195204

29. Pal S, Mukherjee S, Ghosh S (2014) Nonlinear kinetic analysis of phenol adsorption onto peat soil. Environ Earth Sci 71(4):1593-1603 
30. Park Y, Ayoko GA, Frost RL (2011) Characterisation of organoclays and adsorption of p-nitrophenol: Environmental application. J Colloid Interface Sci 360(2):440-456

31. Parolo ME, Pettinari GR, Musso TB, Sánchez-Izquierdo MP, Fernández LG (2014) Characterization of organo-modified bentonite sorbents: The effect of modification conditions on adsorption performance. Appl Surf Sci 320:356-363

32. Rowe RK (2005) Long-term performance of contaminant barrier systems. Geotechnique 55(9):631-678

33. SAC (2008) Chemicals-Adsorption-Desorption Using a Batch Equilibrium Method, GBT21851-2008. China Planning Press, Beijing

34. Sanchez LM, Alvarez VA, Ollier RP (2019) Acid-treated Bentonite as filler in the development of novel composite PVA hydrogels. J Appl Polym Sci 136(25):47663

35. Slade PG, Gates WP (2004) The swelling of HDTMA smectites as influenced by their preparation and layer charges. Appl Clay Sci 25(1-2):93-101

36. Sonne AT et al (2018) Linking ecological health to co-occurring organic and inorganic chemical stressors in a groundwater-fed stream system. Sci Total Environ 642:1153-1162

37. Sun W, Xu G, Wei G, Zhang W, Sun D (2021) Effects of ammonium ion and bentonite content on permeability of bentonite-clay mixture.Environmental Earth Sciences, 80(4)

38. Sun Z, Park Y, Zheng S, Ayoko GA, Frost RL (2013) Thermal stability and hot-stage Raman spectroscopic study of Ca-montmorillonite modified with different surfactants: A comparative study. Thermochimica acta 569:151-160

39. TOUZEFOLTZ N, GAGET E (2006) Hydraulic and mechanical behavior of GCLs in contact with leachate as part of a composite liner. Geotext Geomembr 24(3):188-197

40. Vadlamudi S, Mishra AK (2018) Consolidation Characteristics of Sand-Bentonite Mixtures and the Influence of Sand Particle Size. Journal of Hazardous, Toxic, and Radioactive Waste 22(4):06018001

41. Wan Y, Guo D, Hui X, Liu L, Yao Y (2020) Studies on Hydration Swelling and Bound Water Type of Sodium- and Polymer-Modified Calcium Bentonite. Advances in Polymer Technology, 2020: 1-11

42. Wang A et al (2018) Adsorption Properties of $\mathrm{Pb}^{2+}$ by Amino Group's Functionalized Montmorillonite from Aqueous Solutions. Journal of Chemical \& Engineering Data 63(8):2940-2949

43. Wang $T$ et al (2010) Enhancing the sorption capacity of CTMA-bentonite by simultaneous intercalation of cationic polyacrylamide. J Hazard Mater 178(1-3):1078-1084

44. Xi Y, Frost RL, He H, Kloprogge T, Bostrom T (2005) Modification of Wyoming Montmorillonite Surfaces Using a Cationic Surfactant. Langmuir 21(19):8675-8680

45. Xu H et al (2019) Studies on the chemical compatibility of soil-bentonite cut-off walls for landfills. J Environ Manage 237:155-162

46. Xu H, Zhu W, Qian X, Wang S, Fan X (2016) Studies on hydraulic conductivity and compressibility of backfills for soilbentonite cutoff walls. Appl Clay Sci 132-133:326-335

47. Xu L, Zhang M, Zhu L (2014) Adsorption-desorption behavior of naphthalene onto CDMBA modified bentonite: Contribution of the $\pi-\pi$ interaction. Appl Clay Sci 100:29-34

48. Xue Q, Zhan L, Hu L, Du Y (2020) Environmental geotechnics: state-of-the-art of theory, testing and application to practice. China Civil Eng J 53(3):80-94

49. Xue Q, Zhang Q, Liu L (2012) Impact of High Concentration Solutions on Hydraulic Properties of Geosynthetic Clay Liner Materials. Materials 5(11):2326-2341

50. Yang J, Yu K, Liu C (2017) Chromium immobilization in soil using quaternary ammonium cations modified montmorillonite: Characterization and mechanism. J Hazard Mater 321:73-80 
51. Yang Y et al (2021a) Development of novel multifunctional adsorbent by effectively hosting both zwitterionic surfactant and hydrated ferric oxides in montmorillonite. Sci Total Environ 774:144974

52. Yang $Y$ et al (2021b) Development of novel multifunctional adsorbent by effectively hosting both zwitterionic surfactant and hydrated ferric oxides in montmorillonite. Sci Total Environ 774:144974

53. Yang Y, Reddy KR, Du Y, Fan R (2019) Retention of $\mathrm{Pb}$ and $\mathrm{Cr}(\mathrm{VI})$ onto slurry trench vertical cutoff wall backfill containing phosphate dispersant amended Ca-bentonite. Appl Clay Sci 168:355-365

54. Yang YL, Du YJ, Reddy KR, Fan RD (2017) Phosphate-amended sand/Ca-bentonite mixtures as slurry trench wall backfills: Assessment of workability, compressibility and hydraulic conductivity. Appl Clay Sci 142:120-127

55. Ying SC, Schaefer MV, Cock-Esteb A, Li J, Fendorf S (2017) Depth Stratification Leads to Distinct Zones of Manganese and Arsenic Contaminated Groundwater. Environ Sci Technol 51(16):8926-8932

56. Yu C, Liao R, Cai X, Yu X (2019) Sodium polyacrylate modification method to improve the permeant performance of bentonite in chemical resistance. J Clean Prod 213:242-250

57. Zadaka D, Radian A, Mishael YG (2010) Applying zeta potential measurements to characterize the adsorption on montmorillonite of organic cations as monomers, micelles, or polymers. J Colloid Interface Sci 352(1):171-177

58. Zhang W, Qiu Q (2010) Analysis on contaminant migration through vertical barrier walls in a landfill in China. Environ Earth Sci 61(4):847-852

59. Zhao Q, Burns SE (2012) Molecular Dynamics Simulation of Secondary Sorption Behavior of Montmorillonite Modified by Single Chain Quaternary Ammonium Cations. Environ Sci Technol 46(7):3999-4007

60. Zhao Q, Choo H, Bhatt A, Burns SE, Bate B (2017) Review of the fundamental geochemical and physical behaviors of organoclays in barrier applications. Appl Clay Sci 142:2-20

61. Zhou CH, Cun Jun L, Gates WP, Zhu TT, Wei Hua Y (2019) Co-intercalation of organic cations/amide molecules into montmorillonite with tunable hydrophobicity and swellability. Appl Clay Sci 179:105157

62. Zhu J et al (2011) Preparation and characterization of zwitterionic surfactant-modified montmorillonites. J Colloid Interface Sci 360(2):386-392

63. Zhu J, He H, Zhu L, Wen X, Deng F (2005) Characterization of organic phases in the interlayer of montmorillonite using FTIR and 13C NMR. J Colloid Interface Sci 286(1):239-244

64. Zhu L, Zhu R (2008) Surface structure of CTMA+ modified bentonite and their sorptive characteristics towards organic compounds. Colloids Surf, A 320(1-3):19-24

65. Zhu R et al (2010) Structural and sorptive characteristics of the cetyltrimethylammonium and polyacrylamide modified bentonite. Chem Eng J 160(1):220-225

66. Zhu TT et al (2019) Exfoliation of montmorillonite and related properties of clay/polymer nanocomposites. Appl Clay Sci 169:48-66

\section{Figures}



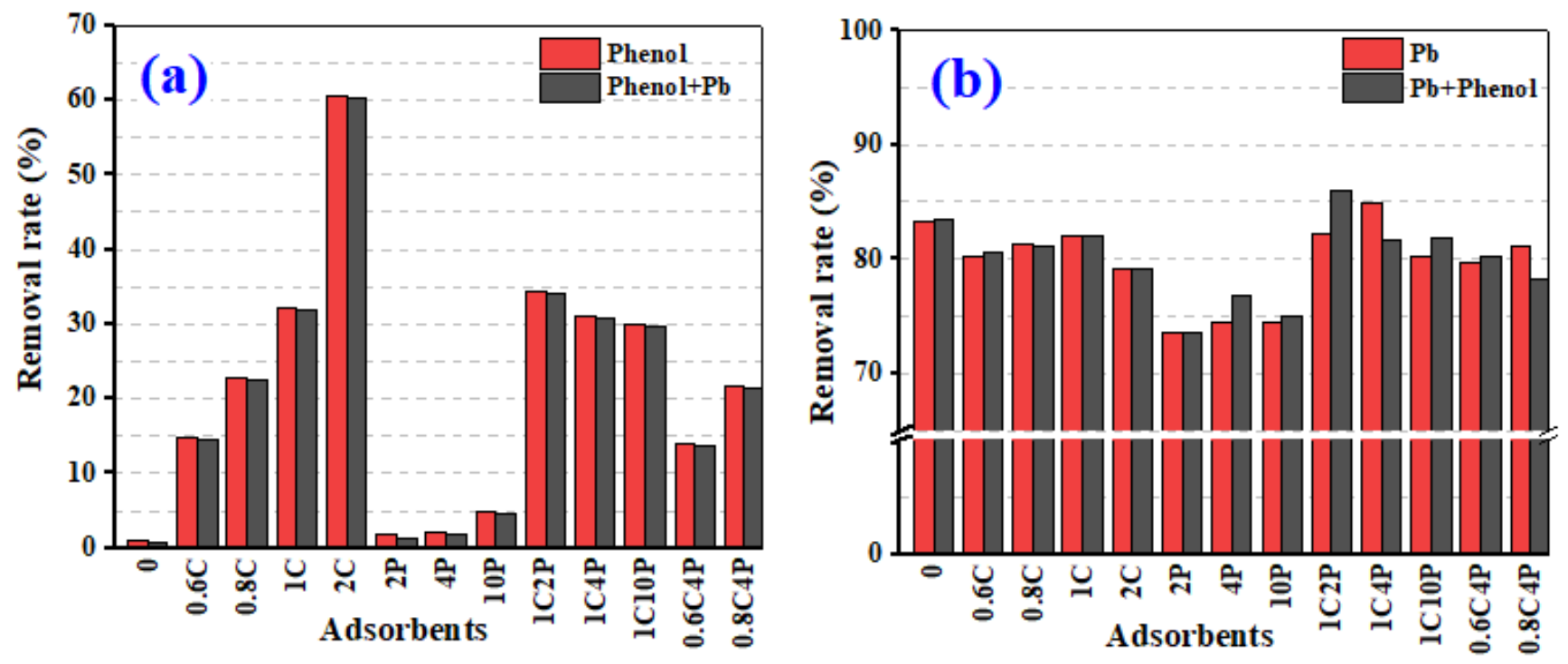

Figure 1

Comparison of adsorption effectiveness of different bentonites on composite and single contaminants: (a) Phenol; (b) $\mathrm{Pb}(\mathrm{II})$.
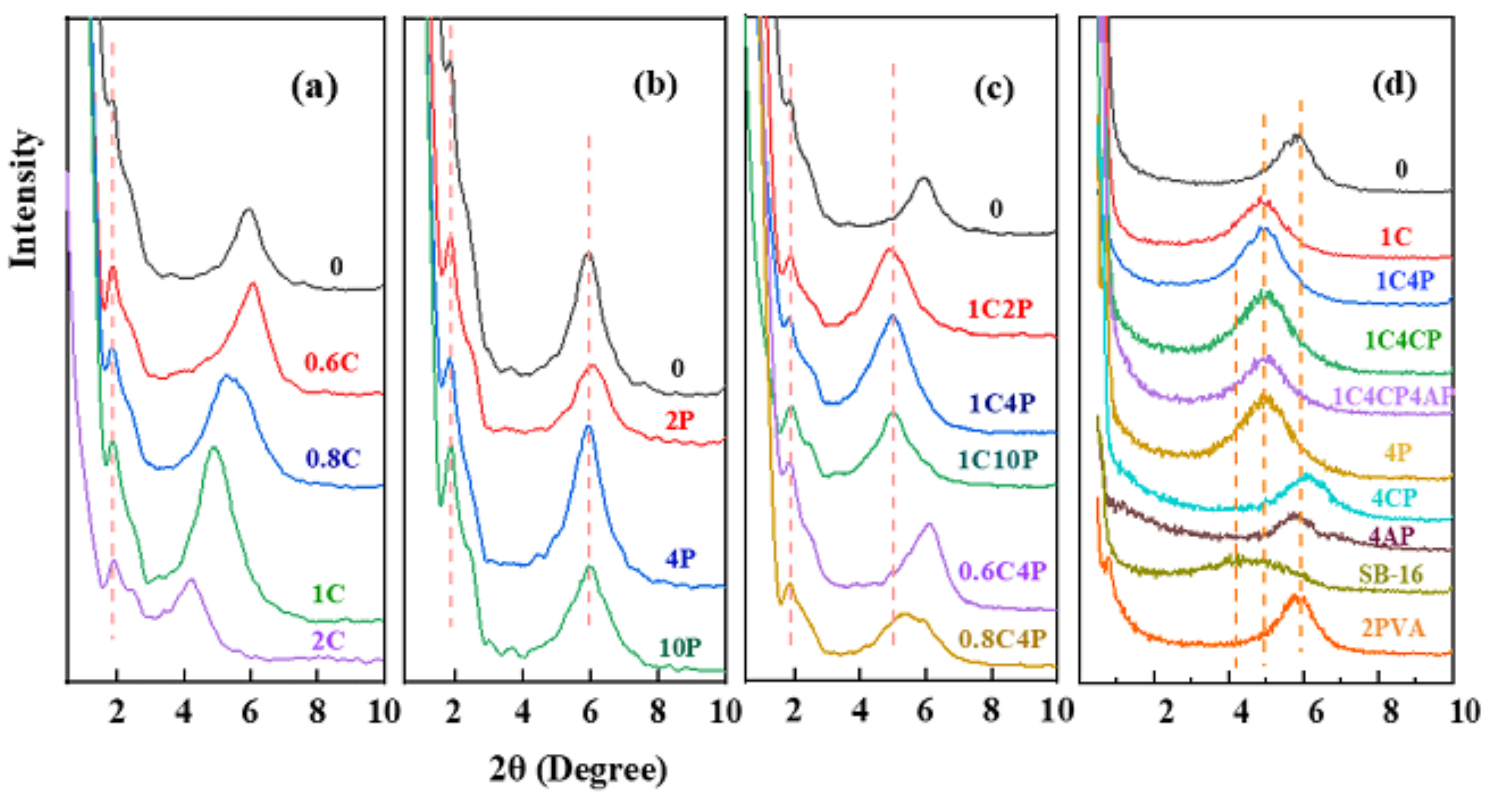

Figure 2

XRD patterns of modified bentonites: (a) CTAB with different concentrations; (b) different types of polymers; (c) composite modification; (d) the samples selected for further studies. 


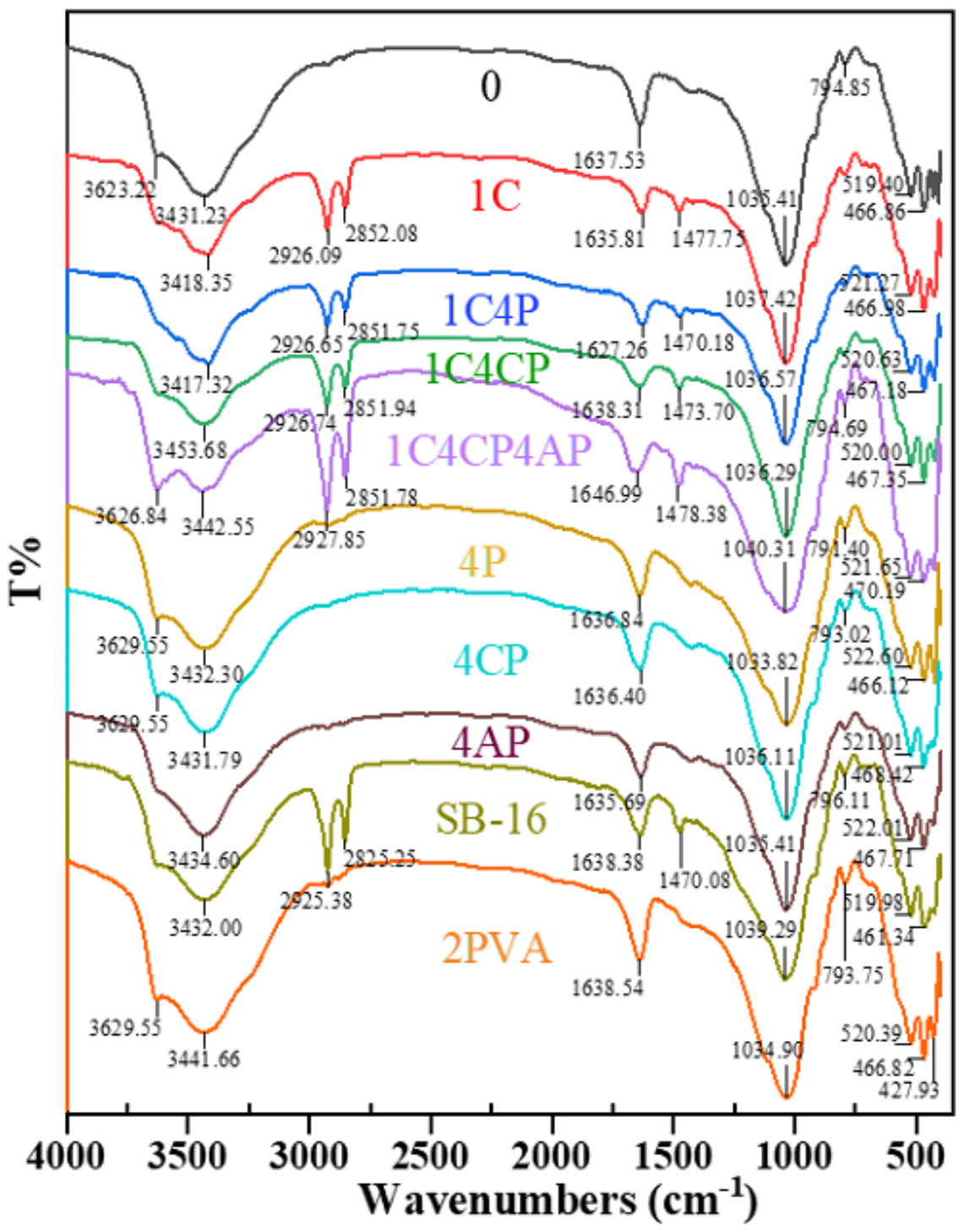

Figure 3

FTIR spectrums of the modified bentonites in the range of $400-4000 \mathrm{~cm}^{-1}$. 


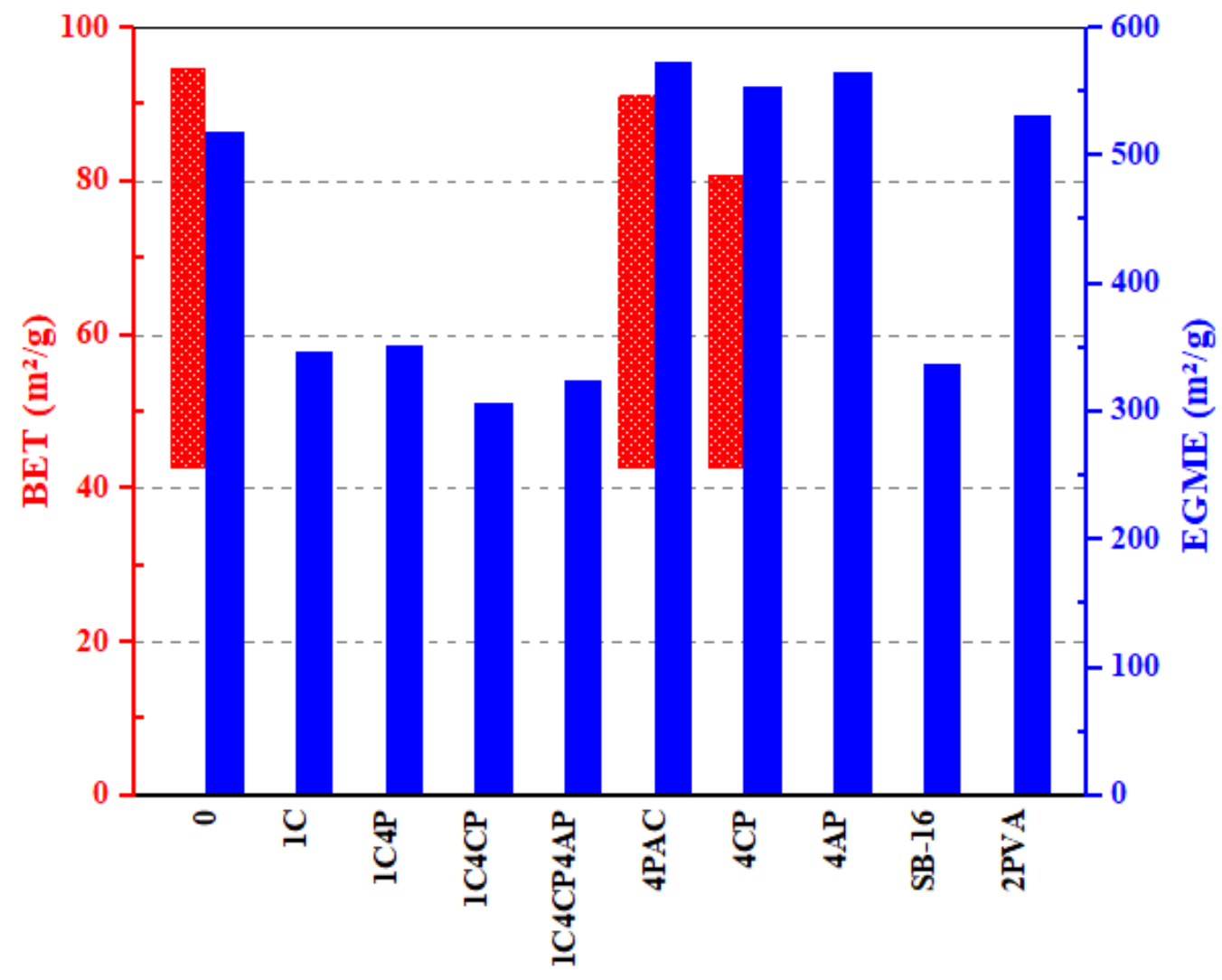

Figure 4

Comparison of specific surface area of modified bentonites obtained with BET and EGME methods.

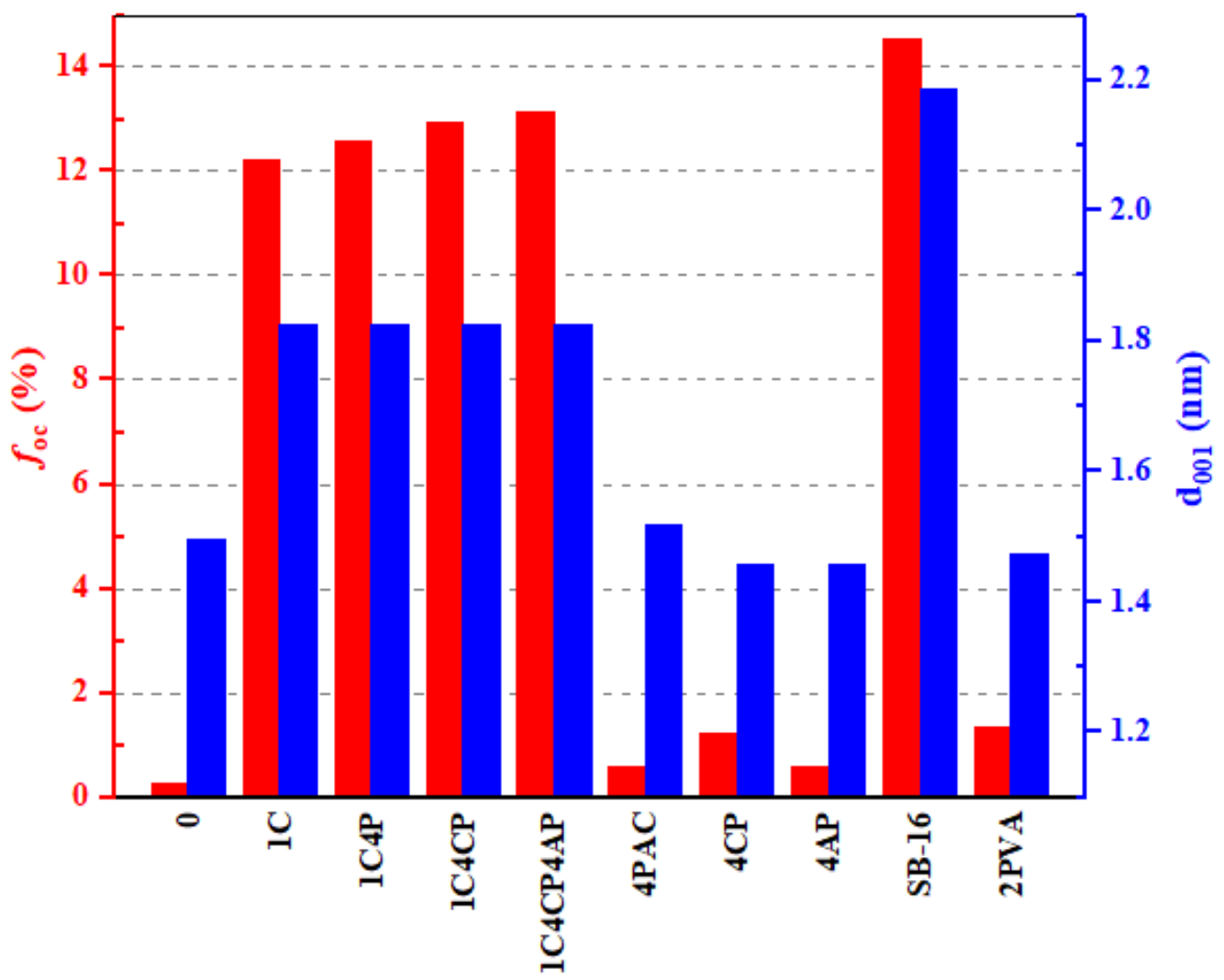


Figure 5

Organic carbon content $\left(f_{\mathrm{oc}}\right)$ and basal spacing $\left(\mathrm{d}_{001}\right)$ of different modified bentonites.

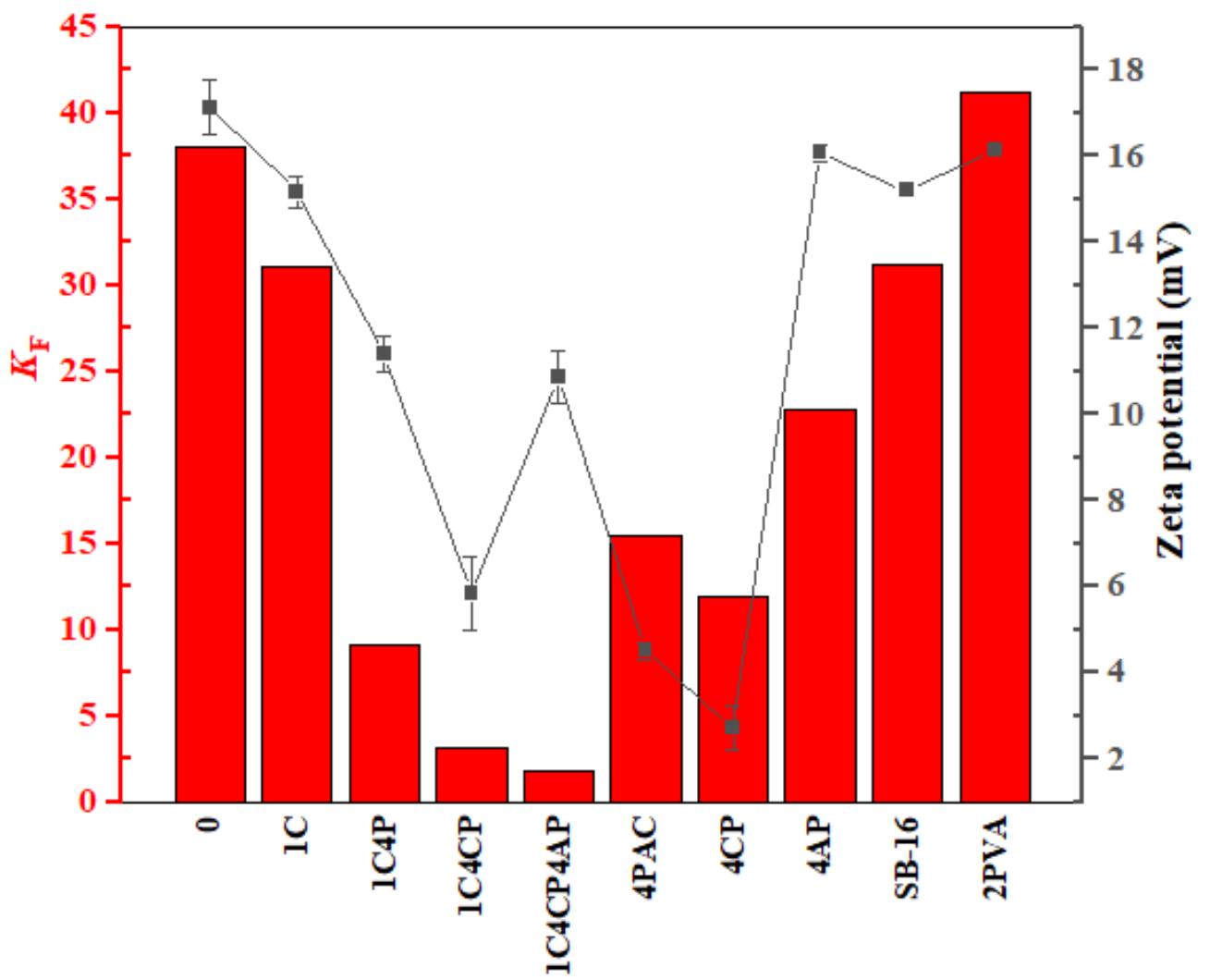

Figure 6

The relationship between $K_{\mathrm{F}}$ and zeta potential. 

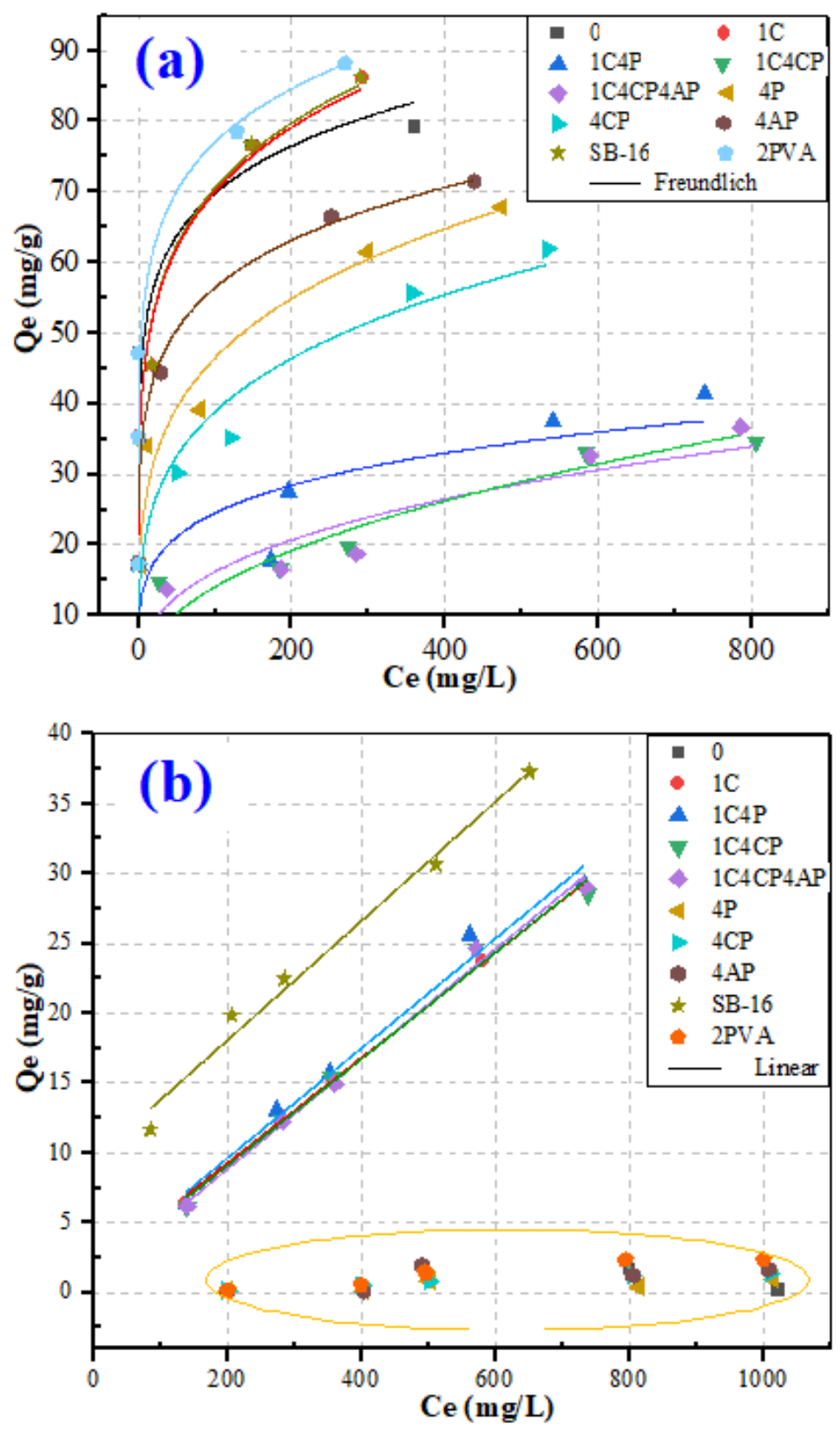

Figure 7

Adsorption isotherms of contaminants on the bentonites, (a) $\mathrm{Pb}(\mathrm{II})$; (b) Phenol. 


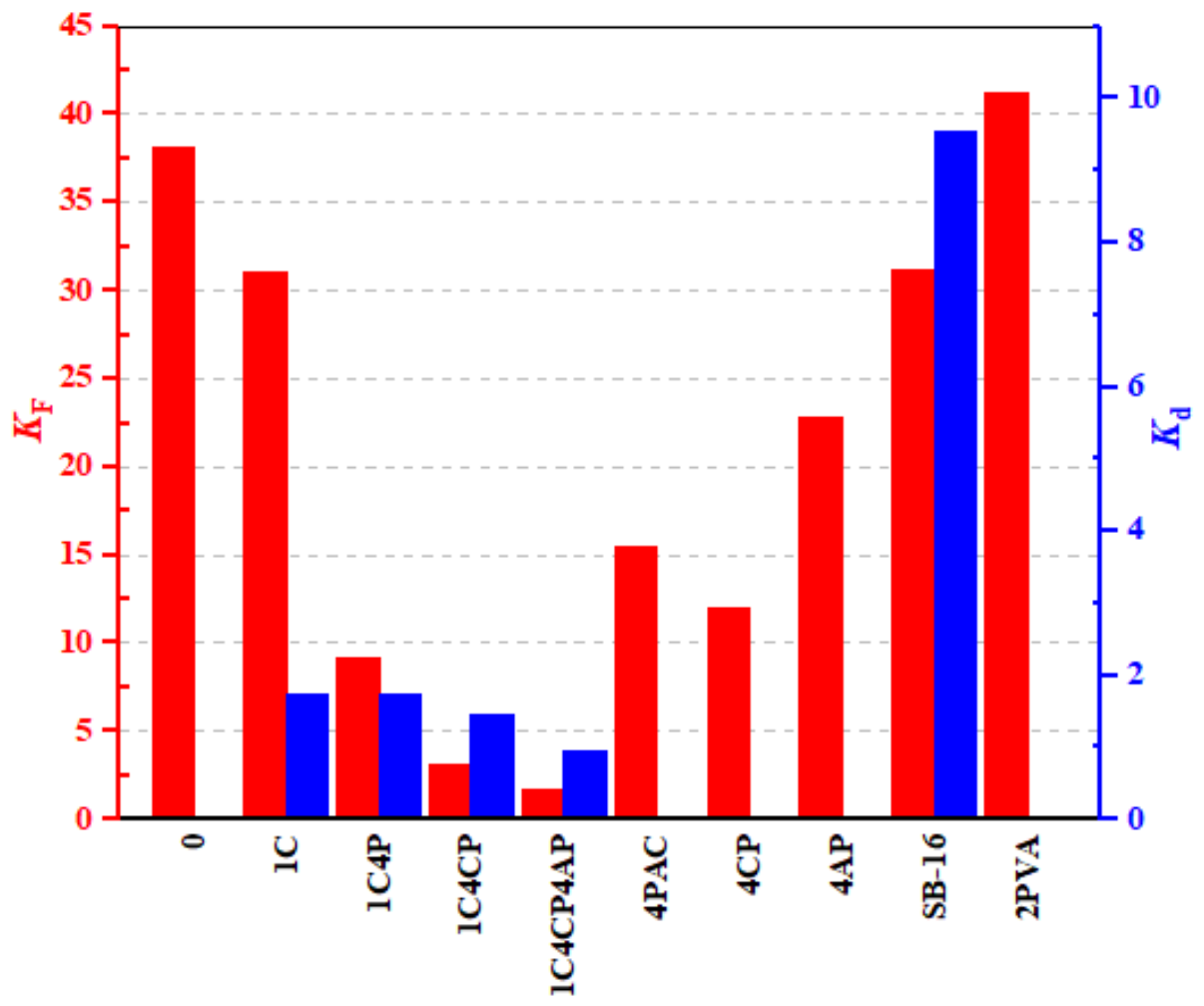

Figure 8

Comparison of adsorption constants of modified bentonites for composite contaminants ( $\mathrm{Pb}(\mathrm{II})$ and Phenol).

\section{Supplementary Files}

This is a list of supplementary files associated with this preprint. Click to download.

- floatimage1.png 